\title{
Identifying Problematic Substance Use in a National Sample of Adolescents Using Frequency Questions
}

\author{
Laura J. Chavez, PhD, MPH, Katharine A. Bradley, MD, MPH, \\ Gwen T. Lapham, PhD, MPH, MSW, Thomas M. Wickizer, PhD, MPH, and \\ Deena J. Chisolm, $P h D$
}

Background: Brief substance use screening questions for tobacco, alcohol, cannabis, and other drugs need further validation in adolescents. In particular, optimal age-specific screening cut-points are not known, and no study has been large enough to evaluate screening questions for noncannabis illicit drug use.

Methods: Adolescent respondents to an annual national household survey were included (2008 to 2014; $n=169,986$ ). Days of tobacco use in the past month, and days of alcohol, cannabis, other illicit drug use in the past year, were assessed as brief screens for tobacco dependence and DSM-IV alcohol (AUD), cannabis (CUD), and other illicit drug use disorders (DUD). Areas under receiver operating characteristics curves (AUCs), sensitivity and specificity were estimated separately by age group (1215-, 16-17-, and 18-20-year-olds) and cut-points that maximized combined values of sensitivity and specificity were considered optimal.

Results: The prevalence of tobacco dependence, AUD, CUD, and DUD was $5.8 \%, 7.1 \%, 4.5 \%$, and $2.0 \%$, respectively. AUCs ranged 0.84 to 0.99 . The optimal cut-points for screening for tobacco dependence and DUDs was the same for all age groups: $\geq 1$ day. The optimal cut-points for alcohol and cannabis varied by age: $\geq 3$ days for $12-15$-year-olds and $\geq 12$ days for older adolescents.

Conclusions: Brief measures of past-year use, or past-month use for tobacco, accurately identified adolescents with problematic substance use. However, health systems should use age-specific screening cut-points for alcohol and cannabis to optimize screening performance. ( $\mathrm{J}$ Am Board Fam Med 2019;32: 550-558.)

Keywords: Adolescent, Cannabis, Substance-Related Disorders, Surveys and Questionnaires, Street Drugs, Tobacco

Use of alcohol and other drugs increases adolescents' risk for altered brain development, cognitive decline, poor school performance, sexual risk behaviors, future substance use disorders, and injury or death. ${ }^{1-5}$ Past-month use of alcohol and other drugs is common, with nearly $33 \%$ of 12 th graders reporting alcohol use, $23 \%$ reporting cannabis use

This article was externally peer reviewed.

Submitted 21 September 2018; revised 8 March 2019; accepted 10 March 2019.

From the College of Public Health, Division of Health Services Management and Policy, Ohio State University, Columbus, OH (LJC, TMW, DJC); Nationwide Children's Hospital, Research Institute, Columbus (LJC, DJC); Kaiser Permanente Washington Health Research Institute, Seattle, WA (KAB, GTL); College of Medicine, Department of Pediatrics, Ohio State University, Columbus (DJC).
(6\% using daily), 24\% reporting illicit drug use, and $11 \%$ reporting cigarette use. ${ }^{6}$ Reducing alcohol, cannabis, other illicit substances, and tobacco use among adolescents is a prevention priority ${ }^{7}$ and family physicians can play an important role in identifying early use and curbing harmful effects. ${ }^{8}$ Although adolescents are often open to discussing

Funding: LJC and other authors did not receive a specific grant for this project from funding agencies in the public, commercial, or not-for-profit sectors. KAB's work on this project was supported by the National Institute of Alcohol Abuse and Alcoholism (K24 AA022128).

Conflict of interest: none declared.

Author contributions: All authors contributed significantly to the work reported in this manuscript, reviewed the final version and approved of its submission.

Corresponding author: Laura J. Chavez, PhD, MPH, College of Public Health, Division of Health Services Management and Policy, Ohio State University, 1841 Neil Ave, Columbus, Ohio 43210 (E-mail:laura.chavez@nationwidechildrens.org). 
substance use with a trusted primary care clinician, ${ }^{9}$ some clinicians cite time barriers for universal screening. ${ }^{10}$ To more widely implement screening for substance use among adolescents, clinicians need brief, valid, screening instruments that can be used quickly and efficiently.

A number of screening tools have been validated to assess substance use among adolescents, ${ }^{11-20}$ varying in their length and complexity, and whether they were developed specifically for adolescents. For family physicians who treat adolescents and adults, deciding whether to use an adolescent-specific screen (such as the CRAFFT screening interview), ${ }^{12,13}$ must be weighed against the ease of using brief, scaled substance-use screens that are effective in adults $^{21,22}$ as well as adolescents. ${ }^{15,17,18,20}$ Such screens require only a few minutes to complete and allow family physicians to use the same screen for all patients, but may require age-specific screening thresholds for adolescents. Use of age-specific screening thresholds may improve performance for brief alcohol screens, ${ }^{14,15,23}$ but it is unknown whether this is true for other substances. Previous validation studies in clinical samples had small numbers of adolescents reporting use of illicit substances other than marijuana. ${ }^{18,20}$ As a result, it remains unknown whether age- or gender- specific screening thresholds are needed, and if so, for which types of substances.

The present study evaluates optimal age- and gender-specific cut-points for brief tobacco, alcohol, cannabis, and other illicit drug screens in adolescents (12 to 20 years) using a large national dataset from the 2008 to 2014 National Survey on Drug Use and Health (NSDUH). ${ }^{24}$ Specifically, the objectives were to assess the screening performance (sensitivity, specificity, and receiver operating characteristics area under the curve [AUC] $)^{25,26}$ of brief frequency-based screening questions against different problematic substance use criterion standards, which included tobacco dependence, meeting DSM-IV diagnostic criteria (alcohol, cannabis or other illicit drugs), or having any symptom of a DSM-IV substance use disorder. In addition, to evaluate whether substance use screens perform differently in the subset of adolescents who see a health care provider, the study evaluates the brief screens for substance use among adolescents reporting a past-year health care visit.

\section{Methods}

\section{Data Source and Sample}

The present study used pooled survey data from the 2008 to 2014 NSDUH. The Substance Abuse and Mental Health Services Administration ${ }^{27}$ sponsors yearly NSDUH collection among the civilian, noninstitutionalized US population, 12 years of age or older. ${ }^{28}$ The study employed an independent, multistage area probability sample design of all 50 states, and the District of Columbia, oversampling adolescents (12 to 17 years) and young adults (18 to 25 years). ${ }^{28}$ The survey assessed tobacco, alcohol, cannabis, and other illicit drug use, mental health conditions, health care utilization, and demographic characteristics. Participants were interviewed in person, but questions were self administered on laptop computers through computerassisted interviewing and interviewers are blinded to participant responses. The study sample included adolescents aged 12 to 20 years, who responded to a NSDUH survey between 2008 to $2014(\mathrm{n}=169,986)$. This age range is similar to that used for adolescents in Bright Futures Guidelines (11 to 21 years), ${ }^{29}$ except for exclusion of the youngest adolescents (age 11 years) who were not eligible for the NSDUH and those age 21 years who were of US legal age for alcohol, and in some states, marijuana consumption. The present study used publicly available data and was determined to be exempt from human subjects review by the Institutional Review Board at Nationwide Children's Hospital.

\section{Measures \\ Brief Screens for Substance Use}

Reported number of days of tobacco, alcohol, cannabis, and other drug use were evaluated as brief screening questions. The NSDUH evaluated smoking frequency in a past-month time frame, asking how many days an adolescent had smoked "part or all a cigarette." However, frequency of alcohol, cannabis, and other illicit drug use were evaluated in a past-year time frame, consistent with other prior validated screening instruments in adolescent samples. ${ }^{12,18,20}$ Specifically, the NSDUH asked respondents how many days they "had a drink of an alcoholic beverage during the past 12 months" and how many days they "used marijuana or hashish during the past 12 months." Similarly, respondents were asked on how many days they 
used multiple other types of illicit substances including cocaine (as well as different forms of cocaine such as "crack"), heroin, hallucinogens, inhalants, prescription pain relievers, tranquilizers, stimulants, and sedatives (nonmedical use without a doctor's prescription, if applicable). For alcohol, cannabis, and other illicit substances, respondents can report past-year frequency as the total days per year, average days per month, or average days per week. The present study used the NSDUH's variable for total number of days per year, in which self-reported average weekly or monthly days of use were multiplied by 52 and 12, respectively.

To approximate a single-item screening question for illicit substances (other than cannabis) used in prior studies, ${ }^{18,20}$ we made a composite measure of the total number of past-year days of illicit substances used across all noncannabis illicit substances. Our composite measure for the total days of "other illicit drug use" was constructed by summing each respondent's number of past-year days of use across all types of noncannabis illicit substances. Because individuals may have used more than 1 type of illicit substance on the same day, a small minority of adolescent respondents' total number of days exceeded 365 days when all types of illicit substances were combined $(\mathrm{n}=572,0.03 \%)$. However, an alternative measure of past-year days of illicit drug use (excluding cannabis) was also constructed for sensitivity analyses. This alternate measure used the greatest number of days of use reported for any 1 illicit substance as the total days of use, assuming that days of all other illicit drug use overlapped with that of the most frequently used substance.

The NSDUH uses a sophisticated imputation procedure to provide imputed values of substance use frequency when responses are missing, and these imputed values were used in primary analyses following recommendations for appropriate analysis of NSDUH data. ${ }^{30}$ However, unimputed frequency of substance use for all substances was also used in sensitivity analyses. The proportions of the total sample with imputed frequency of use data varied by substance and are the following: $2.17 \%$ for alcohol, $1.23 \%$ for cannabis, $2.89 \%$ for other illicit drugs, and $0.11 \%$ for tobacco.

\section{Criterion Standards}

Substance Use Disorders. Respondents reporting past-month cigarette use were asked to respond to the Nicotine Dependence Syndrome Scale ${ }^{31}$ and the Fagerström Test of Nicotine Dependence. ${ }^{32}$ If respondents met criteria for tobacco dependence based on their responses to either the Nicotine Dependence Syndrome Scale or the Fagerström Test of Nicotine Dependence scale they were considered to have past-month tobacco use disorder (TUD). Respondents who reported any alcohol, cannabis, or other illicit drug use were asked to report whether they had experienced any of the 11 substance use disorder symptoms used under the Diagnostic Statistical Manual, fourth edition (DSM-IV). Consistent with DSM-IV, respondents who met either criteria for past-year abuse ( $\geq 1$ of 4 abuse symptoms) or dependence ( $\geq 3$ of 7 dependence symptoms) were considered to have alcohol (AUD), cannabis (CUD), and other illicit drug use disorders (DUD).

Any Substance Use Disorder Symptom. For alcohol, cannabis, and other illicit drug use, a more inclusive criterion standard was evaluated, which included any symptomatic substance use (1 or more DSM-IV symptom of either abuse or dependence).

Outpatient Subgroup. A subgroup of adolescents who reported at least 1 outpatient visit were identified based on the NSDUH survey item, which asked, "During the past 12 months, how many times have you visited a doctor, nurse, physician assistant, or nurse practitioner about your own health at a doctor's office, a clinic, or some other place?" ( $\mathrm{n}=31,505)$. This survey item was added to the NSDUH in 2013 and the outpatient subgroup is limited to 2013 to 2014 surveys. Adolescents with a past-year outpatient health care visit are likely to be of interest to clinicians, given that not all adolescents in the general US population use outpatient health services. ${ }^{33}$

\section{Analyses}

Descriptive statistics were used to characterize the study sample in both the total sample and outpatient subgroup. For each substance use screen (tobacco, alcohol, cannabis, and other illicit drug use), analyses evaluated the sensitivity, specificity and receiver operating characteristics (ROC) area under the curve (AUC) compared with each relevant criterion standard (1 for tobacco; 2 for alcohol, cannabis, and other illicit drug use). The screening cut-points for past month tobacco smoking were $\geq 1$ day, $\geq 2$ days, $\geq 4$ days, $\geq 25$ days. The screen- 
Table 1. Adolescent Sample Characteristics, by Age Group

\begin{tabular}{|c|c|c|c|c|c|c|c|c|}
\hline & \multicolumn{2}{|c|}{$\begin{array}{l}\text { Age } 12 \text { to } 15 \text { Years } \\
\quad \mathrm{N}=79,170\end{array}$} & \multicolumn{2}{|c|}{$\begin{array}{c}\text { Age } 16 \text { to } 17 \text { Years } \\
N=42,356\end{array}$} & \multicolumn{2}{|c|}{$\begin{array}{c}\text { Age } 18 \text { to } 20 \text { Years } \\
N=48,460\end{array}$} & \multicolumn{2}{|c|}{$\begin{array}{c}\text { Total } \\
\mathrm{N}=169,986\end{array}$} \\
\hline & $\mathrm{N}$ & $(\%)$ & $\mathrm{N}$ & $(\%)$ & $\mathrm{N}$ & $(\%)$ & $\mathrm{N}$ & (\%) \\
\hline \multicolumn{9}{|l|}{ Gender } \\
\hline Males & 40,375 & $(51.2)$ & 21,531 & $(50.9)$ & 23,901 & $(51.7)$ & 85,807 & $(51.2)$ \\
\hline Females & 38,795 & $(48.8)$ & 20,825 & $(49.1)$ & 24,559 & $(48.3)$ & 84,179 & $(48.7)$ \\
\hline \multicolumn{9}{|l|}{ Race/ethnicity } \\
\hline White Non-Hispanic & 45,509 & $(56.1)$ & 24,450 & $(57.1)$ & 27,670 & $(56.7)$ & 97,629 & $(56.5)$ \\
\hline Black Non-Hispanic & 10,543 & $(14.2)$ & 5998 & $(14.8)$ & 6863 & $(14.5)$ & 23,404 & $(14.5)$ \\
\hline Hispanic & 15,053 & $(21.5)$ & 7762 & $(20.2)$ & 9180 & $(20.8)$ & 31,995 & $(21.0)$ \\
\hline Asian & 3027 & $(4.9)$ & 1644 & $(5.0)$ & 2120 & $(5.1)$ & 6791 & $(5.0)$ \\
\hline Other & 5038 & (3.3) & 2502 & $(2.9)$ & 2627 & $(2.7)$ & 10,167 & (3.0) \\
\hline \multicolumn{9}{|l|}{ Criterion Standards } \\
\hline Tobacco Dependence & 999 & $(1.1)$ & 2588 & $(5.2)$ & 6250 & $(11.8)$ & 9837 & $(5.8)$ \\
\hline AUD & 1,781 & $(2.1)$ & 3314 & $(7.3)$ & 6490 & $(13.1)$ & 11,585 & $(7.1)$ \\
\hline Any AUD Symptom & 3796 & $(4.5)$ & 7264 & $(16.5)$ & 14,094 & $(28.8)$ & 25,154 & $(15.7)$ \\
\hline CUD & 1509 & $(1.7)$ & 2733 & $(6.1)$ & 3324 & $(6.9)$ & 7566 & $(4.5)$ \\
\hline Any CUD Symptom & 2892 & (3.4) & 5727 & $(12.9)$ & 8534 & $(17.8)$ & 17,153 & $(10.5)$ \\
\hline DUD & 976 & $(1.1)$ & 1004 & $(2.3)$ & 1483 & $(3.0)$ & 3463 & $(2.0)$ \\
\hline Any DUD Symptom & 2114 & $(2.5)$ & 2043 & $(4.7)$ & 2890 & $(5.8)$ & 7047 & $(4.2)$ \\
\hline
\end{tabular}

AUD, alcohol use disorder; CUD, cannabis use disorder; DUD, other illicit drug use disorder.

Ns are unweighted, proportions are weighted to account for complex survey design.

$\chi^{2}$ test comparing Criterion Standards across age groups all $P<.001$.

ing cut-points for alcohol, cannabis, and other drugs were selected based on prior validation studies: $\geq 1$ day of use in the past year, $\geq 2$ days, $\geq 3$ days, $\geq 12$ days, $\geq 52$, $\geq 270$ days. $^{15,18,20}$ Sensitivity and specificity were calculated for each cut-point using weighted proportions with svy commands in Stata 14.0. ${ }^{34}$ ROC curves, which plot sensitivity on the $\mathrm{Y}$-axis and 1-Specificity on the $\mathrm{X}$-axis for the criterion standard, ${ }^{26}$ were plotted for each brief screen and AUC were estimated using PROC SURVEYLOGISTIC and ROC commands in SAS Version 9.4. ${ }^{35}$ Results were stratified by age-group (12 to 15 years, 16 to 17 years, and 18 to 20 years) and secondary analyses additionally stratified by gender. $\chi^{2}$ tests for independence were used to evaluate differences between AUC across subgroups based on age and gender.

Screening thresholds that "balance sensitivity and specificity," or maximize the combined values of sensitivity and specificity are presented. ${ }^{36}$ All analyses accounted for complex survey design. Sensitivity analyses were also conducted using the alternate definition of other illicit drug use. Analyses were repeated for the subgroup of adolescents who reported having at least 1 outpatient visit in the past year, stratifying by age. This secondary analysis was conducted to evaluate whether results differed in the subset of adolescents who use outpatient health care. Sensitivity analyses were also conducted using the alternate definition of frequency of other illicit drug use and using unimputed values of substance use frequency.

\section{Results}

Among 169,986 adolescents in the study sample (age 12 to 15 years, $\mathrm{n}=79,170$; age 16 to 17 years, $\mathrm{n}=42,356$; age 18 to 20 years, $\mathrm{n}=48,460$ ), AUD was the most prevalent substance use disorder (7.1\%), followed by TUD (5.8\%), CUD (4.5\%), and other illicit DUD (2.0\%) (Table 1). An estimated $15.7 \%$ of youth had at least 1 DSM-IV AUD symptom, followed by $10.5 \%$ for CUD symptoms and $4.2 \%$ for DUD symptoms. The prevalence of TUDs, AUDs, CUDs, DUDs, as well as any DSM-IV symptoms of AUDs, CUDs, and DUDs, varied by age and gender (Table 1, Appendix 1). Overall, the prevalence was lowest among 12-15year-olds and increased with age. Comparing females to males, females ages 12 to 15 years had a higher prevalence of both AUD and AUD symptoms, as well as other illicit DUD and DUD symp- 
Table 2. Performance of Substance Use Screens for Identifying Tobacco Dependence and Alcohol, Cannabis, and Other Illicit Drug Use Disorder

\begin{tabular}{|c|c|c|c|c|c|c|}
\hline & \multicolumn{2}{|c|}{$\begin{array}{l}\text { Age } 12 \text { to } 15 \text { Years } \\
\quad \mathrm{N}=79,170\end{array}$} & \multicolumn{2}{|c|}{$\begin{array}{l}\text { Age } 16 \text { to } 17 \text { Years } \\
\qquad N=42,356\end{array}$} & \multicolumn{2}{|c|}{$\begin{array}{l}\text { Age } 18 \text { to } 20 \text { Years } \\
\qquad N=48,460\end{array}$} \\
\hline & Se & $\mathrm{Sp}$ & Se & $\mathrm{Sp}$ & $\mathrm{Se}$ & $\mathrm{Sp}$ \\
\hline \multicolumn{7}{|l|}{ Smoking* } \\
\hline$\geq 1$ day & 1.00 & 0.97 & 1.00 & 0.90 & 1.00 & 0.79 \\
\hline$\geq 2$ days & 0.91 & 0.98 & 0.96 & 0.92 & 0.97 & 0.82 \\
\hline$\geq 4$ days & 0.81 & 0.99 & 0.89 & 0.94 & 0.94 & 0.86 \\
\hline$\geq 25$ days & 0.48 & 1.00 & 0.68 & 0.98 & 0.80 & 0.95 \\
\hline AUC (SE) & \multicolumn{2}{|c|}{$0.99(0.0002)$} & \multicolumn{2}{|c|}{$0.98(0.0003)^{\ddagger}$} & \multicolumn{2}{|c|}{$0.96(0.0004)^{\ddagger \S}$} \\
\hline \multicolumn{7}{|l|}{$\mathrm{Alcohol}^{\dagger}$} \\
\hline$\geq 1$ day & 1.00 & 0.85 & 1.00 & 0.57 & 1.00 & 0.38 \\
\hline$\geq 2$ days & 1.00 & 0.88 & 1.00 & 0.62 & 1.00 & 0.41 \\
\hline$\geq 3$ days & 1.00 & 0.90 & 1.00 & 0.66 & 1.00 & 0.44 \\
\hline$\geq 12$ days & 0.88 & 0.95 & 0.92 & 0.80 & 0.96 & 0.60 \\
\hline$\geq 52$ days & 0.49 & 0.98 & 0.58 & 0.92 & 0.73 & 0.79 \\
\hline$\geq 270$ days & 0.04 & 1.00 & 0.03 & 1.00 & 0.04 & 0.99 \\
\hline AUC (SE) & \multicolumn{2}{|c|}{$0.97(0.0004)$} & \multicolumn{2}{|c|}{$0.91(0.0008)^{\ddagger}$} & \multicolumn{2}{|c|}{$0.84(0.0010)^{\neq \S}$} \\
\hline \multicolumn{7}{|l|}{ Cannabis $^{\dagger}$} \\
\hline$\geq 1$ day & 1.00 & 0.94 & 1.00 & 0.79 & 1.00 & 0.71 \\
\hline$\geq 2$ days & 1.00 & 0.95 & 1.00 & 0.82 & 1.00 & 0.73 \\
\hline$\geq 3$ days & 1.00 & 0.96 & 1.00 & 0.83 & 1.00 & 0.75 \\
\hline$\geq 12$ days & 0.93 & 0.97 & 0.95 & 0.88 & 0.97 & 0.81 \\
\hline$\geq 52$ days & 0.66 & 0.99 & 0.74 & 0.93 & 0.83 & 0.87 \\
\hline$\geq 270$ days & 0.14 & 1.00 & 0.20 & 0.98 & 0.36 & 0.96 \\
\hline AUC (SE) & \multicolumn{2}{|c|}{$0.99(0.0002)$} & \multicolumn{2}{|c|}{$0.95(0.0006)^{\ddagger}$} & \multicolumn{2}{|c|}{$0.93(0.0007)^{\ddagger \S}$} \\
\hline \multicolumn{7}{|c|}{ Other Illicit Drugs $^{\dagger}$} \\
\hline$\geq 1$ day & 1.00 & 0.93 & 1.00 & 0.88 & 1.00 & 0.84 \\
\hline$\geq 2$ days & 0.95 & 0.94 & 0.97 & 0.90 & 0.98 & 0.87 \\
\hline$\geq 3$ days & 0.92 & 0.95 & 0.93 & 0.92 & 0.96 & 0.88 \\
\hline$\geq 12$ days & 0.75 & 0.97 & 0.80 & 0.95 & 0.85 & 0.93 \\
\hline$\geq 52$ days & 0.48 & 0.98 & 0.57 & 0.98 & 0.64 & 0.97 \\
\hline$\geq 270$ days & 0.14 & 1.00 & 0.19 & 1.00 & 0.26 & 1.00 \\
\hline AUC (SE) & \multicolumn{2}{|c|}{$0.98(0.0004)$} & \multicolumn{2}{|c|}{$0.97(0.0005)^{\ddagger}$} & \multicolumn{2}{|c|}{$0.97(0.0005)^{\ddagger \S}$} \\
\hline
\end{tabular}

AUC, Area under Receiver Operating Characteristic (ROC) Curve for Tobacco Dependence (Nicotine Dependence Syndrome Scale/Fagerström Test of Nicotine Dependence) or DSM-IV Alcohol, Cannabis, or Other Drug Use Disorders; Se, Sensitivity; Sp, Specificity.

Bolded screening cut-points are shown that have high values of sensitivity and specificity and minimize the number of different ageand substance-specific cut-points.

*Frequency is coded as days of use in the past month.

${ }^{\dagger}$ Frequency is coded as days of use in the past year.

$\chi^{2}$ test for independence (relative to age 12 to 15 years), ${ }^{\ddagger} P<.0001$.

$\chi^{2}$ test for independence (comparing 16-17- and 18-20-year-olds), ${ }^{\circledR} P<.0001$.

toms, but not CUD, CUD symptoms, or tobacco dependence (Appendix 1). However, among 1820-year-olds, males had a higher prevalence than females of all substance use disorders and symptoms, as well as tobacco dependence.

\section{Substance Use Disorder as Criterion Standard}

Reported number of days of substance use had high AUCs for identifying TUD, AUD, CUD, and
DUD (AUCs, 0.84 to 0.99) (Table 2). AUCs were significantly lower among each older age group (18 to 20 years and 16 to 17 years) relative to $12-15$ year-olds $(P$-values all $<.01)$ (Table 2; Appendix $2)$. For TUD, AUCs were significantly lower among males age 16 to 17 years and 18 to 20 years, relative to females $(P<.001)$ (Appendix 3). For AUD, AUCs were significantly lower among males 
18 to 20 years relative to females $(P<.001)$, but did not differ for other age groups. For other DUD, AUCs were significantly lower for males compared with females for ages 12 to 15 years and 18 to 20 years $(P$ values both $<.001)$, but not for ages 16 to 17 years. However, AUCs did not differ significantly by gender for CUD in any age group.

Reporting $\geq 1$ day of past-month smoking balanced sensitivity and specificity for identifying tobacco dependence among all age groups (Table 2). For both AUD and CUD, the cut-points that balanced sensitivity and specificity were $\geq 3$ days of past-year use for $12-15$-year-olds and $\geq 12$ days for 16-17- and 18-20-year-olds. For other illicit DUD, the cut-points that balanced sensitivity and specificity were $\geq 1$ day among $12-15$ - and 16-17year-olds, but was slightly higher ( $\geq 2$ days) among 18-20-year-olds, though. However, a cut-point of $\geq 1$ day of illicit drug use also performed well among 18-20-year-olds (sensitivity and specificity $>0.80$ ). Cut-points that balanced sensitivity and specificity were similar for female and male adolescents (Appendix 3). Analyses that evaluated screening cut-points of past-year days based on the alternate definition of days of other illicit drug use did not meaningfully change from our main results (data not shown). In addition, when analyses were repeated using unimputed substance use frequency values, the screening cut-points did not change.

\section{Any Substance Use Disorder Symptom as Criterion Standard}

Analyses using a more inclusive criterion standard of 1 or more DSM-IV substance use disorder symptoms (AUD, CUD, or other DUD) in the total sample produced similar results to those that used DSM-IV substance use disorder (Appendix 4, Appendix 5).

\section{Outpatient Subgroup}

Overall, $73.4 \%$ of adolescents age 12 to 20 years in 2013 to 2014 reported any past-year outpatient visit and were included in the outpatient subgroup $(\mathrm{n}=31,505)$ (Appendix 6). Compared with the total sample, similar cut-points for balancing sensitivity and specificity were found for all criterion standards among the outpatient subgroup (Appendix 7, Appendix 8). All brief substance use screens were also associated with high values of AUCs $(0.83$ to 0.99$)$.

\section{Discussion}

This study evaluated brief screens that ask about the frequency of use of tobacco, alcohol, cannabis, and other illicit drugs in a large national sample of adolescents, allowing adequate samples to evaluate optimal screening cut-points across different age and gender subgroups. The substance use screens performed well for identifying AUD, CUD, DUD, and TUD, and screens for alcohol, cannabis, and other illicit drugs performed well for identifying adolescents with 1 or more DSM-IV symptom of AUD, CUD, or DUD. Optimal cut-points that balanced sensitivity and specificity did not differ by gender, but did differ by age-group for alcohol and cannabis. These findings suggest that brief screens using frequency of substance use like those used in adults are effective in adolescents, but that screening cut-points should be varied across age groups for some substances.

The present study confirms the validity of single-item screens for TUD, AUD, and CUD, while providing important information on optimal cutpoints. Frequency of past-year tobacco use has been evaluated as a single-item screen in adolescent samples, ${ }^{18,20}$ but this is the first study to confirm that any past-month use had high sensitivity and specificity for identifying TUD and that recommended cut-points did not vary by gender or agegroup. Several studies have evaluated age-specific differences in a single-item screen for AUD, ${ }^{14,15,23}$ but no prior study has simultaneously estimated age-specific screening cut-points for single-item screens for other types of substances in the same adolescent sample. Only 2 prior studies evaluated past-year frequency of marijuana use as a brief screen, ${ }^{18,20}$ both included adolescents age 12 to 17 years, but neither evaluated age-specific cut-points. Although screening thresholds differed by agegroup for both AUD and CUD in our sample, the cut-points that optimized screening performance in each age-group were the same for both substances (both $\geq 3$ days for age 12 to 15 years; $\geq 12$ days for 16 to 17 and 18 to 20 years). Finally, this study is the first to document that any past-year days of other illicit drug use ( $\geq 1$ days) had high sensitivity and specificity for identifying DUD (both $>0.80$ ) across all age groups.

Selection of screening thresholds typically seeks to strike a balance between sensitivity and specificity, but must also consider resources available for 
Table 3. Single-Item Screens for Identifying Substance Use Disorder (or Tobacco Dependence) in Adolescents (Age 12 to 20 years)

Screening Questions Screening Cut-Points for Positive Screen

1. During the past 30 days, on how many days did you smoke part or all of a cigarette?

2. On how many days in the past 12 months did you drink an alcoholic beverage?

$\geq 1$ day all age groups

3. On how many days in the past 12 months did you use marijuana or hashish?

$\geq 3$ days for ages 12 to 15 years

$\geq 12$ days if older

3. On how many days in the past 12 months did you use marijuana or hashish?

4. On how many days in the past 12 months did you use cocaine, crack, heroin, hallucinogens, inhalants, or prescription medications including pain relievers, tranquilizers, stimulants, or sedatives that were not prescribed for you or that you took only for the feeling that it caused.

Screening cut-points are shown that have high values of sensitivity and specificity and minimize the number of different age- and substance-specific cut-points.

screening. Based on the results of this study, brief substance use frequency questions could be used to screen adolescents. We recommend some screening cut-points that achieved high sensitivity and specificity in our sample, but minimized the number of different age- and substance-specific cut-points (Table 3). If health systems use electronic self-administered screening, or pen and paper screens that are entered into electronic health records (EHRs), ageand substance-specific cut-points could be programmed into tablets, kiosks, or EHRs. However, in systems relying on clinician interview screening these cut-points may need further simplification. Clinicians could consider using the cut-point that balanced sensitivity and specificity for identifying AUD and CUD among the youngest age-group ( $\geq 3$ days of past-year use) for all ages. The decisions on which screening threshold to use will need to be made while keeping in mind the goals and costs of screening.

Information on adolescent substance use can inform all aspects of primary care for adolescent patients. Validated screens for substance use are superior to relying on clinical impressions for identifying adolescents with problematic substance use. ${ }^{37}$ In addition to triggering assessments for symptoms of substance use disorders, information about substance use could affect treatment plans or the type of follow-up and monitoring that is warranted. For example, adolescents with chronic conditions who drink alcohol have lower medication adherence compared with those who do not drink, ${ }^{38}$ and may need closer monitoring to ensure that their chronic conditions are being managed. Alcohol can also interact with many commonly prescribed medications, ${ }^{39}$ and clinicians may need to discuss these adverse outcomes with adolescent patients who have chronic conditions, many of whom may be unaware of the risks of medication interactions. Finally, substance use behaviors in adolescents often occurs with other adverse health behaviors, such as sexual risk behaviors and psychosocial problems. ${ }^{1,3}$ Addressing these issues early has the potential to prevent or reduce adverse consequences of continued substance use.

There are limitations to the present study. First, this study used DSM-IV criteria as the criterion standard. NSDUH has not yet transitioned to using DSM-5 criteria, which are now used in clinical settings. DSM-5 criteria no longer distinguish between abuse and dependence and the prevalence of substance use disorders may differ from that under DSM-IV. ${ }^{40}$ However, a recent study that validated the CRAFFT screen among adolescents using DSM-5 criteria found the thresholds for positive screens remained the same as those validated using DSM-IV. ${ }^{13}$ Second, adolescents' reports of substance use and related problems on NSDUH were confidential, and it is unknown how this may affect responses. However, all validation studies of substance use screening tools among adolescents, to our knowledge, have used similar conditions of anonymity (screening results were not shared with health care providers or parents). ${ }^{12,13,16,18,20} \mathrm{Un}$ der-reporting may be more common in clinical settings where substance use is shared with clinicians or documented in the medical record if adolescents fear their substance use may be disclosed to parents. Clinicians will likely need to build rapport and emphasize the confidentiality of their health 
information in order for adolescents to feel comfortable disclosing substance use. ${ }^{41}$

\section{Conclusions}

Single-item screens for substance use are often used in adults. The present study found that brief single-item screens using reported number of days of use were effective for identifying adolescents with tobacco, alcohol, cannabis, and other illicit drug use disorders, irrespective of age-group. These findings suggest that efforts to integrate behavioral health into primary care settings could use the same screens for adults and adolescents. However, optimal screening cut-points varied by age groups for alcohol and cannabis. Self-administered screening using tablets or kiosks, or article questionnaire entered into EHRs, could optimize screening by using these age-specific screening thresholds. Alternatively, a single screening threshold for each substance with high sensitivity in all age groups could be used in systems where it is not feasible to use age-specific cut-points.

To see this article online, please go to: http://jabfm.org/content/ 32/4/550.full.

\section{References}

1. Windle M. Drinking over the lifespan: Focus on early adolescents and youth. Alcohol Res 2016;38: 95-101.

2. Dawson DA, Goldstein RB, Chou SP, et al. Age at first drink and the first incidence of adult-onset DSM-IV alcohol use disorders. Alcohol Clin Exp Res 2008;32:2149-2160.

3. Maggs JL, Staff J, Kloska DD, et al. Predicting young adult degree attainment by late adolescent marijuana use. J Adolesc Health 2015;57:205-211.

4. Volkow ND, Compton WM, Weiss SR. Adverse health effects of marijuana use. N Engl J Med 2014; 371:879.

5. Murray CJ, Atkinson C, Bhalla K, et al. The state of US health, 1990-2010: burden of diseases, injuries, and risk factors. JAMA 2013;310:591-608.

6. National Institute on Drug Abuse. Monitoring the future study: Trends in prevalence of various drugs. Available from: https://www.drugabuse.gov/trendsstatistics/monitoring-future/monitoring-future-studytrends-in-prevalence-various-drugs. Accessed July 5, 2017.

7. Koh HK, Blakey CR, Roper AY. Healthy People 2020: A report card on the health of the nation. JAMA 2014;311:2475-2476.

8. Griswold KS, Aronoff H, Kernan JB, et al. Adolescent substance use and abuse: Recognition and management. Am Fam Physician 2008;77:331-336.
9. Yoast RA, Fleming M, Balch GI. Reactions to a concept for physician intervention in adolescent alcohol use. J Adolesc Health 2007;41:35-41.

10. Sterling S, Kline-Simon AH, Wibbelsman C, et al. Screening for adolescent alcohol and drug use in pediatric health-care settings: Predictors and implications for practice and policy. Addict Sci Clin Pract 2012;7:13.

11. Knight JR, Sherritt L, Harris SK, et al. Validity of brief alcohol screening tests among adolescents: A comparison of the AUDIT, POSIT, CAGE, and CRAFFT. Alcohol Clin Exp Res 2003;27:67-73.

12. Knight JR, Sherritt L, Shrier LA, et al. Validity of the CRAFFT substance abuse screening test among adolescent clinic patients. Arch Pediatr Adolesc Med 2002;156:607-614.

13. Mitchell SG, Kelly SM, Gryczynski J, et al. The CRAFFT cut-points and DSM-5 criteria for alcohol and other drugs: A reevaluation and reexamination. Subst Abus 2014;35:376-380.

14. Chung T, Colby SM, Barnett NP, et al. Screening adolescents for problem drinking: Performance of brief screens against DSM-IV alcohol diagnoses. J Stud Alcohol 2000;61:579-587.

15. Clark DB, Martin CS, Chung T, et al. Screening for underage drinking and Diagnostic and Statistical Manual of Mental Disorders, 5 th Edition alcohol use disorder in rural primary care practice. J Pediatr 2016;173:214-220.

16. Gryczynski J, Kelly SM, Mitchell SG, et al. Validation and performance of the Alcohol, Smoking and Substance Involvement Screening Test (ASSIST) among adolescent primary care patients. Addiction 2015;110:240-247.

17. Levy S, Dedeoglu F, Gaffin JM, et al. A screening tool for assessing alcohol use risk among medically vulnerable youth. PloS One 2016;11:e0156240.

18. Levy S, Williams JF. An electronic screen for triaging adolescent substance use by risk levels. JAMA Pediatr 2014;168:822-828.

19. Kelly TM, Donovan JE, Chung T, et al. Brief screens for detecting alcohol use disorder among 18-20 year old young adults in emergency departments: Comparing AUDIT-C, CRAFFT, RAPS4QF, FAST, RUFT-Cut, and DSM-IV 2-Item Scale. Addict Behav 2009;34:668-674.

20. Kelly SM, Gryczynski J, Mitchell SG, et al. Validity of brief screening instrument for adolescent tobacco, alcohol, and drug use. Pediatrics 2014;133:819-826.

21. McNeely J, Strauss SM, Saitz R, et al. A brief patient self-administered substance use screening tool for primary care: Two-site validation Study of the Substance Use Brief Screen (SUBS). Am J Med 2015; 128:784 e789-e719.

22. Smith PC, Schmidt SM, Allensworth-Davies D, et al. A single-question screening test for drug use in primary care. Arch Intern Med 2010;170:1155-1160. 
23. Parast L, Meredith LS, Stein BD, et al. Identifying adolescents with alcohol use disorder: Optimal screening using the National Institute on Alcohol Abuse and Alcoholism screening guide. Psychol Addict Behav 2018;32:508-516.

24. Substance Abuse and Mental Health Services Administration. National Survey on Drug Use and Health. 2019. Available from: https://www.samhsa. gov/data/data-we-collect/nsduh-national-survey-druguse-and-health. Accessed January 25, 2019.

25. Koepsell T, Weiss, NS. Measurement error. In: Epidemiologic methods: Studying the occurrence of illness. New York, NY: Oxford University Press; 2003, 223-229.

26. Zweig MH, Campbell G. Receiver-operating characteristic (ROC) plots: A fundamental evaluation tool in clinical medicine. Clin Chem 1993;39:561577.

27. Substance Abuse and Mental Health Services Administration. Available from: https://www.samhsa. gov. Accessed January 25, 2019.

28. Center for Behavioral Health Statistics and Quality. 2014 National Survey on Drug Use and Health: Methodological Resource Book (Section 2, Sample Design Report). Rockville, MD: Substance Abuse and Mental Health Services Administration; 2015.

29. Hagan J, Shaw JS, Duncan PM, eds. Bright futures: Guidelines for health supervision of infants, children, and adolescents. 4th ed. Elk Grove Village, IL: American Academy of Pediatrics; 2017.

30. Center for Behavioral Health Statistics and Quality. 2014 National Survey on Drug Use and Health: Methodological resource book (Section 10: Editing and imputation report). Rockville, MD: Substance Abuse and Mental Health Services Administration; 2016.

31. Shiffman S, Waters A, Hickcox M. The nicotine dependence syndrome scale: A multidimensional measure of nicotine dependence. Nicotine Tob Res 2004;6:327-348.

32. Heatherton TF, Kozlowski LT, Frecker RC, et al. The Fagerström Test for Nicotine Dependence: A revision of the Fagerström Tolerance Questionnaire. Br J Addict 1991;86:1119-1127.

33. Black LI, Nugent CN, Vahratian A. Access and utilization of selected preventive health services among adolescents aged 10-17. NCHS Data Brief 2016; 246:1-8.

34. StataCorp. Stata Statistical Software: Release 14. College Station, TX. StataCorp LP; 2015.

35. SAS Institute Inc., Copyright 2002-2015, Cary, NC.

36. Dawson DA, Grant BF, Stinson FS, et al. Effectiveness of the derived Alcohol Use Disorders Identification Test (AUDIT-C) in screening for alcohol use disorders and risk drinking in the US general population. Alcohol Clin Exp Res 2005;29:844-854.

37. Wilson CR, Sherritt L, Gates E, et al. Are clinical impressions of adolescent substance use accurate? Pediatrics 2004;114(5):e536-e540.

38. Weitzman ER, Ziemnik RE, Huang Q, et al. Alcohol and marijuana use and treatment nonadherence among medically vulnerable youth. Pediatrics 2015; 136:450-457.

39. National Institute on Alcohol Abuse and Alcoholism. Harmful interactions, mixing alcohol with medicines. 2014. NIH Publication No. 13-5329.

40. Center for Behavioral Health Statistics and Quality. Impact of the DSM-IV to DSM-5 Changes on the National Survey of Drug Use and Health. In. Susbtance Abuse and Mental Health Services Administration, Rockville, MD, 2016.

41. Levy SJ, Williams JF. Substance use screening, brief intervention, and referral to treatment. Pediatrics 2016;138(1):e20161211. 